\title{
Self-Consistent Vertex Correction Analysis for Iron-Based Superconductors: Mechanism of Coulomb-Interaction-Driven Orbital Fluctuations
}

\author{
Seiichiro ONARI ${ }^{1}$ and Hiroshi KONTANI ${ }^{2}$ \\ ${ }^{1}$ Department of Applied Physics, Nagoya University and JST, TRIP, Furo-cho, Nagoya 464-8602, Japan. \\ ${ }^{2}$ Department of Physics, Nagoya University and JST, TRIP, Furo-cho, Nagoya 464-8602, Japan.
}

(Dated: October 31, 2018)

\begin{abstract}
We study the mechanism of orbital/spin fluctuations due to multiorbital Coulomb interaction in iron-based superconductors, going beyond the random-phase-approximation. For this purpose, we develop a self-consistent vertex correction (SC-VC) method, and find that multiple orbital fluctuations in addition to spin fluctuations are mutually emphasized by the "multimode interference effect" described by the VC. Then, both the antiferro-orbital and ferro-orbital (=nematic) fluctuations simultaneously develop for $J / U \sim 0.1$, both of which contribute to the $s$-wave superconductivity. Especially, the ferro-orbital fluctuations give the orthorhombic structure transition as well as the softening of shear modulus $C_{66}$.
\end{abstract}

PACS numbers: 74.70.Xa, 74.20.-z, 74.20.Rp

Since the discovery of iron-based superconductors, the mechanism of high- $T_{\mathrm{c}}$ superconductivity has been studied very actively. Theoretically, both the spin-fluctuationmediated $s_{ \pm}$-wave state (with sign reversal of the gap between hole-pocket (h-pocket) and electron-pocket (epocket)) [1-5] and the orbital-fluctuation-mediated $s_{++^{-}}$ wave state (without sign reversal) $[6,7]$ had been proposed. The latter scenario is supported by the robustness of $T_{\mathrm{c}}$ against impurities in many iron-pnictides [812]. Possibility of impurity-induced crossover from $s_{ \pm}$to $s_{++}$states had been discussed theoretically $[3,6]$. Also, orbital-independent gap observed in $\mathrm{BaFe}_{2}(\mathrm{As}, \mathrm{P})_{2}$ and $(\mathrm{K}, \mathrm{Ba}) \mathrm{Fe}_{2} \mathrm{As}_{2}$ by laser ARPES measurement $[13,14]$ as well as the "resonance-like" hump structure in the neutron inelastic scattering [15] are consistent with the orbital fluctuation scenario.

Nature of orbital fluctuations has been studied intensively after the discovery of large softening of the shear modulus $C_{66}$ [16-18] and renormalization of phonon velocity [19] observed well above the orthorhombic structure transition temperature $T_{S}$. Consistently, a sizable orbital polarization is observed in the orthorhombic phase $[20,21]$. Moreover, the "electronic nematic state" with large in-plane anisotropy of resistivity or magnetization well above $T_{S}$ and $T_{\mathrm{c}}[22,23]$, also indicates the occurrence of (impurity-induced local) orbital order [24].

Origin of orbital order/fluctuation had been actively discussed, mainly based on the multiorbital Hubbard model with intra (inter) orbital interaction $U\left(U^{\prime}\right)$ and the exchange interaction $J=\left(U-U^{\prime}\right) / 2>0[6,25]$. We had focused attention to a good inter-orbital nesting of the Fermi surfaces shown in Fig. 1 (a): Although moderate orbital fluctuations are induced by $U^{\prime}$ in the randomphase-approximation (RPA), the spin susceptibility due to the intra-orbital nesting, $\chi^{s}(\boldsymbol{q})$, is the most divergent for $J>0$ (i.e., $U>U^{\prime}$ ). Since $J / U \approx 0.12-0.15$ according to the first-principle study [26], the RPA fails to explain experimental "nonmagnetic" structure transition.
This situation is unchanged even if the self-energy correction is considered in the fluctuation-exchange (FLEX) approximation [27].

To explain the strong development of orbital fluctuations, we had introduced a quadrupole interaction [6]:

$$
H_{\text {quad }}=-g \sum_{i}\left(\hat{O}_{x z}^{i} \hat{O}_{x z}^{i}+\hat{O}_{y z}^{i} \hat{O}_{y z}^{i}\right)
$$

where $g$ is the coupling constant, and $\hat{O}_{\gamma}$ is the charge quadrupole operator $; \gamma=x z, y z, x y, x^{2}-y^{2}, 3 z^{2}-r^{2}$. (Hereafter, $x, y$-axes ( $X, Y$-axes) are along the nearest $\mathrm{Fe}-\mathrm{Fe}$ (Fe-As) direction.) This term is actually caused by the electron-phonon ( $e$-ph) coupling due to in-plane Fe-ion oscillations $[6,14,27]$. Since $\hat{O}_{x z(y z)}$ induces the inter-orbital scattering, strong antiferro (AF) orbital fluctuations develop for $g \gtrsim 0.2 \mathrm{eV}$ owing to a good interorbital nesting. We also studied the vertex correction (VC) beyond the RPA [28], and obtained strong enhancement of ferro-quadrupole $\left(\hat{O}_{x^{2}-y^{2}} \propto \hat{n}_{x z}-\hat{n}_{y z}\right)$ susceptibility $\chi_{x^{2}-y^{2}}^{c}(\mathbf{0})$, which causes the orthorhombic structure transition and the softening of $C_{66}$ [28]. This "nematic fluctuation" is derived from the interference of two AF orbitons due to the symmetry relation $\hat{O}_{x^{2}-y^{2}}(\mathbf{0}) \sim \hat{O}_{X Z}(\boldsymbol{Q}) \times \hat{O}_{Y Z}(-\boldsymbol{Q})$, where $\hat{O}_{X Z(Y Z)}=$ $\left[\hat{O}_{x z}+(-) \hat{O}_{y z}\right] / \sqrt{2}$. Then, it was natural to expect that such multi-orbiton interference effect, which is given by the VC while dropped in the RPA, induces large "Coulomb-interaction-driven" orbital fluctuations.

In this letter, we study the orbital and spin fluctuations in iron-based superconductors by considering the multiorbital Coulomb interaction with $U=U^{\prime}+2 J$ and $J / U \sim O(0.1)$. We develop the self-consistent-VC (SC$\mathrm{VC})$ method, and find that both ferro- $\mathrm{O}_{x^{2}-y^{2}}$ and AF$O_{x z / y z}$ fluctuations strongly develop even for $J / U \sim 0.1$, due to the inter-orbital nesting and the positive interference between multi-fluctuation (orbiton+magnon) modes. This result leads to a conclusion that RPA underestimates the orbital fluctuations in multiorbital sys- 
tems. The present study offers a unified explanation for both the superconductivity and structure transition in many compounds.

Here, we study the five-orbital Hubbard model introduced in Ref. [1]. We denote $d$-orbitals $m=3 z^{2}-r^{2}$, $x z, y z, x y$, and $x^{2}-y^{2}$ as $1,2,3,4$ and 5 , respectively. The Fermi surfaces are mainly composed of orbitals 2, 3 and 4 [28]. Then, the susceptibility for the charge (spin) channel is given by the following $25 \times 25$ matrix form in the orbital basis:

$$
\hat{\chi}^{c(s)}(q)=\hat{\chi}^{\operatorname{irr}, c(s)}(q)\left(1-\hat{\Gamma}^{c(s)} \hat{\chi}^{\operatorname{irr}, c(s)}(q)\right)^{-1},
$$

where $q=\left(\boldsymbol{q}, \omega_{l}=2 \pi l T\right)$, and $\hat{\Gamma}^{c(s)}$ represents the Coulomb interaction for the charge (spin) channel composed of $U, U^{\prime}$ and $J$ given in Refs. [6, 14]. The irreducible susceptibility in Eq. (2) is given as

$$
\hat{\chi}^{\operatorname{irr}, c(s)}(q)=\hat{\chi}^{0}(q)+\hat{X}^{c(s)}(q),
$$

where $\chi_{l l^{\prime}, m m^{\prime}}^{0}(q)=-T \sum_{p} G_{l m}(p+q) G_{m^{\prime} l^{\prime}}(p)$ is the bare bubble, and the second term is the $\mathrm{VC}$ (or orbiton or magnon self-energy) that is neglected in both RPA and FLEX approximation. In the present discussion, it is convenient to consider the quadrupole susceptibilities:

$$
\begin{aligned}
\chi_{\gamma, \gamma^{\prime}}^{c}(q) & \equiv \sum_{l l^{\prime}, m m^{\prime}} O_{\gamma}^{l, l^{\prime}} \chi_{l l^{\prime}, m m^{\prime}}^{c}(q) O_{\gamma^{\prime}}^{m^{\prime}, m} \\
& =\operatorname{Tr}\left\{\hat{O}_{\gamma} \hat{\chi}^{c}(q) \hat{O}_{\gamma^{\prime}}\right\} .
\end{aligned}
$$

Non-zero matrix elements of the quadrupole operators for the orbital $2 \sim 4$ are $O_{x z}^{3,4}=O_{y z}^{2,4}=O_{x^{2}-y^{2}}^{2,2}=$ $-O_{x^{2}-y^{2}}^{3,3}=1[28]$. Because of the symmetry, the offdiagonal susceptibilities $\left(\gamma \neq \gamma^{\prime}\right)$ are zero or very small for $\boldsymbol{q}=\mathbf{0}$ and the nesting vector $\boldsymbol{Q} \approx(\pi, 0)$ or $\boldsymbol{Q}^{\prime} \approx(0, \pi)$ [28]. We do not discuss the angular momentum (dipole) susceptibility, $\chi_{\mu}^{c}(\boldsymbol{q}) \sim\left\langle\hat{l}_{\mu}(\boldsymbol{q}) \hat{l}_{\mu}(-\boldsymbol{q})\right\rangle$, since it is found to be suppressed by the VC. Note that $\hat{O}_{\mu \nu} \propto \hat{l}_{\mu} \hat{l}_{\nu}+\hat{l}_{\nu} \hat{l}_{\mu}$.

To measure the distance from the criticality, we introduce the charge (spin) Stoner factor $\alpha_{\boldsymbol{q}}^{c(s)}$, which is the largest eigenvalue of $\hat{\Gamma}^{c(s)} \hat{\chi}^{\operatorname{irr}, c(s)}(\boldsymbol{q})$ at $\omega_{l}=0$ : The charge (spin) susceptibility diverges when $\alpha_{\max }^{c(s)} \equiv$ $\max _{\boldsymbol{q}}\left\{\alpha_{\boldsymbol{q}}^{c(s)}\right\}=1$. In a special case $J=0$, the relation $\alpha_{\max }^{s}=\alpha_{\max }^{c}$ holds at the momentum $\boldsymbol{Q}$ in the RPA; see Fig. 1 (b). That is, both spin and orbital susceptibilities are equally enhanced at $J=0$, which is unchanged by the self-energy correction in the FLEX approximation [27]. For $J>0$, the spin fluctuations are always dominant $\left(\alpha_{\max }^{s}>\alpha_{\max }^{c}\right)$ in the RPA or FLEX. However, because of large $\hat{X}^{c}(q)$, the opposite relation $\alpha_{\max }^{s} \lesssim \alpha_{\max }^{c}$ can be realized even for $J / U \lesssim 0.1$ in the SC-VC method.

First, we perform the RPA calculation for $n=6.1$ and $T=0.05$, using $32 \times 32 \boldsymbol{k}$-meshes: The unit of energy is $\mathrm{eV}$ hereafter. Figure 1 (c) shows the diagonal quadrupole susceptibilities for $J / U=0.088 ; \chi_{\gamma}^{c}(q) \equiv \chi_{\gamma \gamma}^{c}(q)$. (The spin susceptibility is shown in Ref. [1].) The Stoner
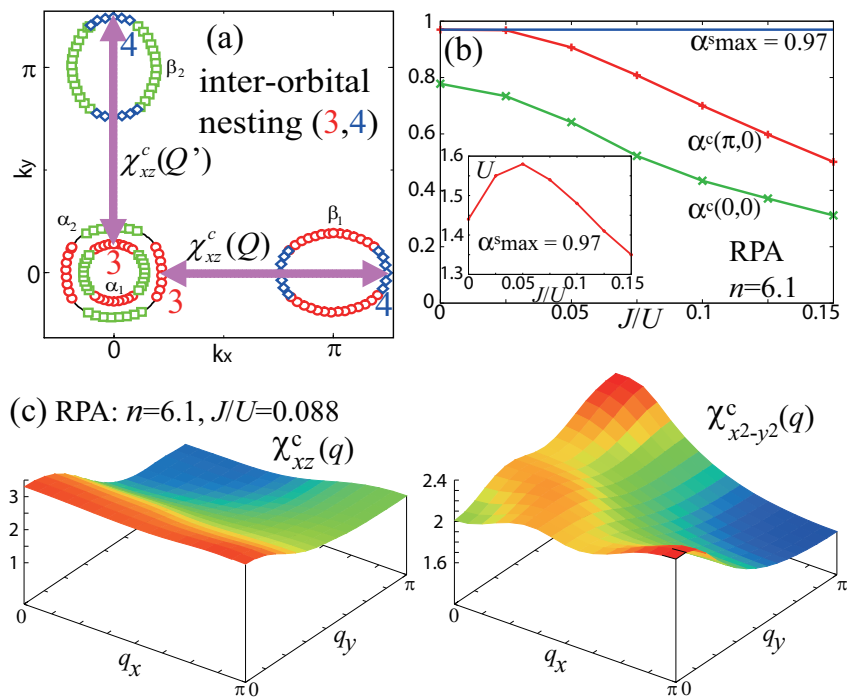

FIG. 1: (color online) (a) Fermi surfaces of iron pnictides. The colors correspond to $2=x z$ (green), $3=y z$ (red), and $4=x y$ (blue), respectively. (b) $\alpha_{\boldsymbol{Q}}^{c}, \alpha_{\mathbf{0}}^{c}$ and $U$ as function of $J / U$ in RPA under the condition $\alpha_{\max }^{s}=0.97$. (c) $\chi_{x z}^{c}(\boldsymbol{q})$ and $\chi_{x^{2}-y^{2}}^{c}(\boldsymbol{q})$ given by the RPA for $(J / U, U)=(0.088,1.53)$.

factors are $\alpha_{\max }^{s}=0.97, \alpha_{Q}^{c}=0.76$, and $\alpha_{0}^{c}=0.47$; see Fig. 1 (b). In the RPA, $\chi_{x z}^{c}(\boldsymbol{Q})\left[\chi_{y z}^{c}\left(\boldsymbol{Q}^{\prime}\right)\right]$ is weakly enlarged by the inter-orbital $(3,4)[(2,4)]$ nesting, while $\chi_{x^{2}-y^{2}}^{c}(\boldsymbol{q})$ is relatively small and AF-like. Thus, the RPA cannot explain the structure transition that requires the divergence of $\chi_{x^{2}-y^{2}}^{c}(\mathbf{0})$.

Next, we study the role of $\mathrm{VC}$ due to the MakiThompson (MT) and Aslamazov-Larkin (AL) terms in Fig. 2 (a), which become important near the critical point $[29,30]$. Here, $\hat{X}^{c(s)}(q) \equiv \hat{X}^{\uparrow, \uparrow}(q)+(-) \hat{X}^{\uparrow, \downarrow}(q)$, and wavy lines represent $\chi^{s, c}$. The AL term (AL1+AL2) for the charge sector, $X_{l l^{\prime}, m m^{\prime}}^{\mathrm{AL}, c}(q)$, is given as

$$
\begin{aligned}
& \frac{T}{2} \sum_{k} \sum_{a \sim h} \Lambda_{l l^{\prime}, a b, e f}(q ; k)\left\{V_{a b, c d}^{c}(k+q) V_{e f, g h}^{c}(-k)\right. \\
& \left.\quad+3 V_{a b, c d}^{s}(k+q) V_{e f, g h}^{s}(-k)\right\} \Lambda_{m m^{\prime}, c d, g h}^{\prime}(q ; k),
\end{aligned}
$$

where $\hat{V}^{s, c}(q) \equiv \hat{\Gamma}^{s, c}+\hat{\Gamma}^{s, c} \hat{\chi}^{s, c}(q) \hat{\Gamma}^{s, c}, \hat{\Lambda}(q ; k)$ is the three-point vertex made of three Green functions in Fig. 2 (a) [28], and $\Lambda_{m m^{\prime}, c d, g h}^{\prime}(q ; k) \equiv \Lambda_{c h, m g, d m^{\prime}}(q ; k)+$ $\Lambda_{g d, m c, h m^{\prime}}(q ;-k-q)$. We include all $U^{2}$-terms, which are important for reliable results. The expressions of other VCs will be published in future.

Both MT and AL terms correspond to the first-order mode-coupling corrections to the RPA susceptibility: The intra- (inter-) bubble correction gives the MT (AL) term [29]. In single-orbital models, the $\mathrm{VC}$ due to $\mathrm{MT}+\mathrm{AL}$ terms had been studied by the self-consistentrenormalization (SCR) theory [29] or FLEX approximation with VC [30], and successful results had been obtained. In the former (latter) theory, the susceptibility is calculated in the self-consistent (self-inconsistent) way. 

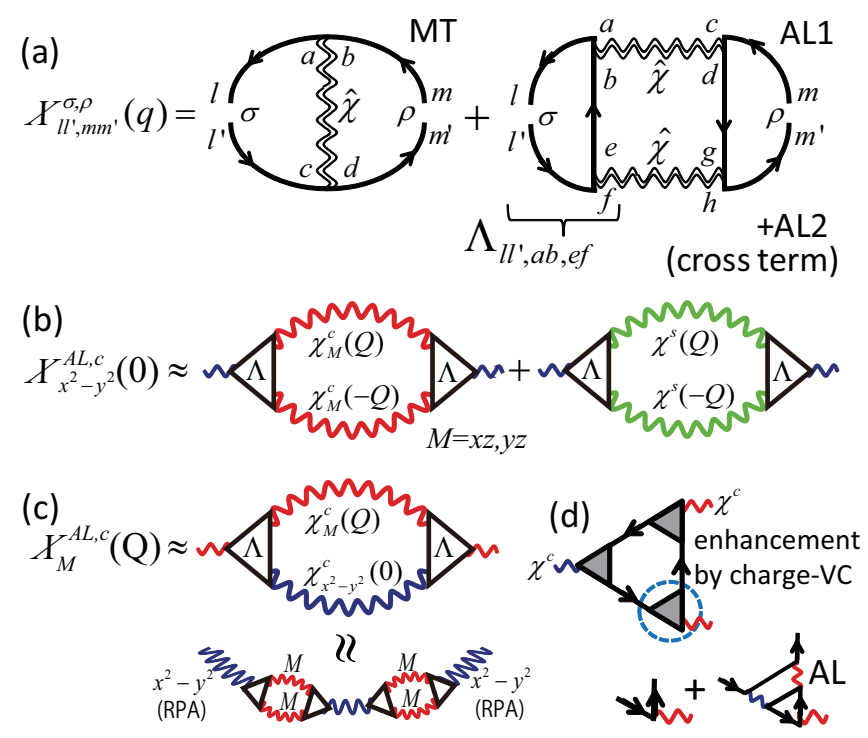

FIG. 2: (color online) (a) The MT and AL terms: The wavy and solid lines are susceptibilities and electron Green functions, respectively. $\Lambda_{l l^{\prime}, a b, e f}$ is the three-point vertex. (b) Dominant AL terms for $\chi_{x^{2}-y^{2}}^{c}(\mathbf{0})$; the first (second) term represents the two-orbiton (two-magnon) process. (c) Dominant AL terms for $\chi_{M}^{c}(\boldsymbol{Q})(M=x z, y z)$; higher-order terms with bubbles made of $\chi_{M}^{c}( \pm \boldsymbol{Q})$ (= multi-fluctuation process) are relevant. (d) Enhancement of $\Lambda_{l l^{\prime}, a b, \text { ef }}$ due to charge VCs.

Here, we find a significant role of the AL term inherent in the multiorbital Hubbard model.

Now, we perform the SC-VC analysis, in the way to satisfy $\hat{\chi}^{c, s}(q)$ in the $\mathrm{VC}$ are equal to the total susceptibilities in Eq. (2). Then, $\hat{\chi}^{c}(q)$ is strongly enhanced by $X^{\mathrm{AL}, c}$ in Eq. (5), which is relevant when either $\hat{\chi}^{c}$ or $\hat{\chi}^{s}$ is large. On the other hand, we have verified numerically that $\hat{X}^{s} \sim T \sum \Lambda \cdot V^{s} V^{c} \cdot \Lambda$ is less important, although it could be relevant only when both $\hat{\chi}^{c}$ and $\hat{\chi}^{s}$ are large. Hereafter, we drop $\hat{X}^{s}(q)$ to simplify the argument. Figure 3 (a) show $\chi_{\gamma}^{c}(\boldsymbol{q})$ given by the SC-VC method for $n=6.1, J / U=0.088$ and $U=1.53$, in which the Stoner factors are $\alpha_{\max }^{s}=\alpha_{\mathbf{0}}^{c}=0.97$ and $\alpha_{\boldsymbol{Q}}^{c}=0.86$. Compared to the RPA, both $\chi_{x^{2}-y^{2}}^{c}(\boldsymbol{q})$ and $\chi_{x z}^{c}(\boldsymbol{q})$ are strongly enhanced by the charge AL term, $\hat{X}^{\mathrm{AL}, c}$, since the results are essentially unchanged even if MT term is dropped. In the SC-VC method, the enhancements of other charge multipole susceptibilities are small. Especially, both the density and dipole susceptibilities, $\sum_{l, m} \hat{\chi}_{l l, m m}^{c}(\boldsymbol{q})$ and $\chi_{\mu}^{c}(q)(\mu=x, y, z)$ respectively, are suppressed.

Here, we discuss the importance of the AL term: At $\boldsymbol{q} \approx \mathbf{0}$ or $\boldsymbol{Q}, \chi_{\gamma}^{c}(\boldsymbol{q})$ is enlarged by the diagonal vertex correction with respect to $\gamma$, $X_{\gamma}^{\mathrm{AL}, c}(\boldsymbol{q}) \equiv \operatorname{Tr}\left\{\hat{O}_{\gamma} \hat{X}^{\mathrm{AL}, c}(\boldsymbol{q}) \hat{O}_{\gamma}\right\} / \operatorname{Tr}\left\{\hat{O}_{\gamma}^{2}\right\}$, since the offdiagonal terms are absent or small [28]. The charge AL term in Eq. (5) is given by the products of two $\chi^{c}$ s (two-orbiton process) and two $\chi^{s}$ s (two-magnon process), shown in Fig. 2 (b). The former process was discussed in Ref. [28], and the latter has a similarity to the spin nematic theory in Ref. [16] based on a frustrated spin model. Now, we consider the orbital selection rule for the two-orbiton process: Because of the relation $\operatorname{Tr}\left\{\hat{O}_{x^{2}-y^{2}} \hat{O}_{M}^{2}\right\} \neq 0$ for $M=x z, y z$ and a rough relation $\Lambda_{l l^{\prime}, a b, c d} \sim \Lambda_{l l^{\prime}, l^{\prime}, b l} \delta_{l^{\prime}, a} \delta_{b, c} \delta_{d, l}$ [28], the two-orbiton process for $\gamma=x^{2}-y^{2}$ is mainly given by $\chi_{M}^{c}(\boldsymbol{Q})^{2}$. According to Eq. (5) and Ref. [28], $X_{x^{2}-y^{2}}^{\mathrm{AL}, c}(\mathbf{0}) \sim \Lambda^{2} U^{4} T \sum_{q}\{\chi(q)\}^{2}$ grows in proportion to $T \chi(\boldsymbol{Q})\left[\log \{\chi(\boldsymbol{Q})\}^{2}\right]$ at high [low] temperatures. In the case of Fig. 3 (a), two-magnon process is more important for $\chi_{x^{2}-y^{2}}^{c}(\mathbf{0})$ because of the relation $\alpha_{\boldsymbol{Q}}^{s}>\alpha_{\boldsymbol{Q}}^{c}$. We checked that the two-magnon process is mainly caused by $\chi_{22,22}^{s}(\boldsymbol{Q})^{2}-\chi_{22,33}^{s}(\boldsymbol{Q})^{2}>0$.

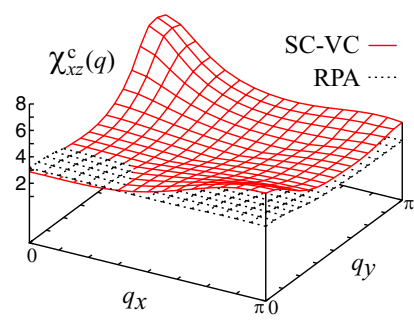

(a) SC-VC: $n=6.1, J / U=0.088$
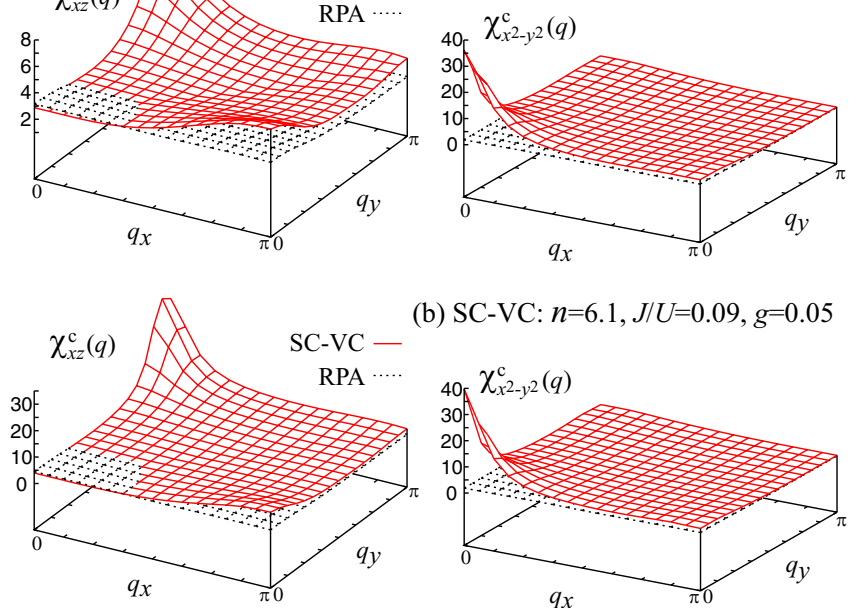

(b) SC-VC: $n=6.1, J / U=0.09, g=0.05$
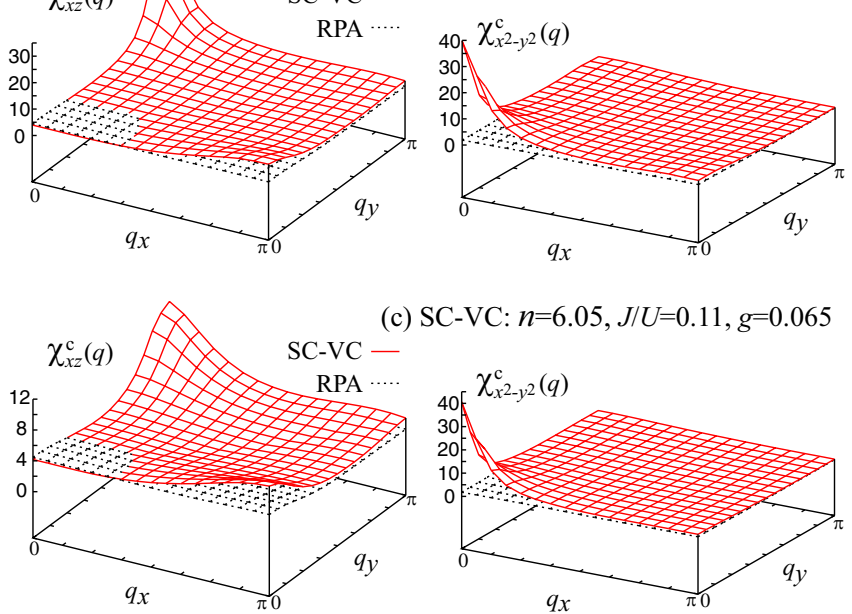

(c) SC-VC: $n=6.05, J / U=0.11, g=0.065$

FIG. 3: (color online) $\chi_{x z}^{c}(\boldsymbol{q})$ and $\chi_{x^{2}-y^{2}}^{c}(\boldsymbol{q})$ given by the SC$\mathrm{VC}$ method. The relation $\alpha_{\max }^{s}=\alpha_{\mathbf{0}}^{c}=0.97$ is satisfied in all cases: (a) $n=6.1$ and $J / U=0.88\left(\alpha_{Q}^{c}=0.86\right)$, (b) $n=6.1$, $J / U=0.9$ and $g=0.05\left(\alpha_{Q}^{c}=0.96\right)$, and (c) $n=6.05$, $J / U=0.11$ and $g=0.065\left(\alpha_{Q}^{c}=0.87\right)$.

In the same way, $X_{M}^{c}(\boldsymbol{Q}) \sim \Lambda^{2} U^{4} T \sum_{q} \chi_{M}^{c}(q+$ $Q) \chi_{x^{2}-y^{2}}^{c}(q)$ is enlarged by the two-orbiton process due to $\chi_{M}^{c}(\boldsymbol{Q})$ and $\chi_{x^{2}-y^{2}}^{c}(\mathbf{0})$, shown in Fig. 2 (c). (In this case, two-magnon process is less important since $\chi^{s}(\mathbf{0})$ is small.) The obtained $\chi_{x z}^{c}(\boldsymbol{q})$ has peaks at $\boldsymbol{q}=\boldsymbol{Q}$ and $\boldsymbol{Q}^{\prime}$ since the inter-orbital scattering is emphasized by $X_{x z}^{c}(\boldsymbol{Q}) \propto \chi_{x^{2}-y^{2}}^{c}(\mathbf{0}) \gg 1$. Thus, both $\chi_{x z}^{c}(\boldsymbol{Q})$ and $\chi_{x^{2}-y^{2}}^{c}(\mathbf{0})$ are strongly enlarged in the SC-VC method, because of the "positive feedback" brought by these two 
AL terms: Figure 2 (c) shows an example of the higherorder terms that are automatically generated in the SCVC method. Such "multi-fluctuation processes" inherent in the self-consistent method magnify the RPA results.

Thus, strong ferro- and AF-orbital fluctuations are caused by AL terms. Both fluctuations work as the pairing interaction for the $s_{++}$-state, while the ferrofluctuations are also favorable for the $s_{ \pm}$-state. For $J / U<(J / U)_{c} \equiv 0.088$, the relation $\alpha_{\max }^{s}<\alpha_{\mathbf{0}}^{c}=0.97$ is realized and $\alpha_{\boldsymbol{Q}}^{c}$ increases towards unity. In this case, orbital order occurs prior to the spin order as increasing $U$ with $J / U$ is fixed, since the $\mathrm{VC}$ (due to two-orbiton process) can efficiently enlarge orbital susceptibilities because of large $\alpha_{\max }^{c}(\mathrm{RPA})$. This situation would be consistent with wider non-magnetic orthorhombic phase in $\mathrm{Nd}(\mathrm{Fe}, \mathrm{Co})$ As and many 1111 compounds.

Since the present SC-VC method is very timeconsuming, we applied some simplifications: We have verified in the self-inconsistent calculation that $\operatorname{Tr}\left\{\hat{O}_{\gamma} \hat{X}(q) \hat{O}_{\gamma^{\prime}}\right\}$ with $\gamma \neq \gamma^{\prime}$ is zero or very small, especially at $\boldsymbol{q}=\mathbf{0}$ and $\boldsymbol{Q}$ for the reason of symmetry. Since we are interested in the enhancement of $\chi_{\gamma}^{c}(\boldsymbol{q})$ at $\boldsymbol{q}=\mathbf{0}$ and $\boldsymbol{Q}$ and the dominant interferences between $\gamma=x z, y z, x^{2}-y^{2}$, we calculated $X_{l l^{\prime}, m m^{\prime}}(q)$ only for $\left\{\left(l, l^{\prime}\right),\left(m, m^{\prime}\right)\right\} \in$ $x z, y z, x^{2}-y^{2} . \quad\left[\left(l, l^{\prime}\right) \in \gamma\right.$ means that $O_{\gamma}^{l, l^{\prime}} \neq$ 0$.$] That is, \left\{\left(l, l^{\prime}\right),\left(m, m^{\prime}\right)\right\}=\{(1,2),(3,4),(2,5)\}$, $\{(1,3),(2,4),(3,5)\}$, and $\{(1,5),(2,2),(3,3)\}$.

We stress that both $(J / U)_{c}$ and AF-orbital fluctuations increase by considering following two factors: The first one is the charge $\mathrm{VC}$ at each point of the threepoint vertex in Fig. 2 (d), as a consequence of the Ward identity between $\hat{\Lambda}$ and $\hat{\chi}^{\text {irr }}$. The enhancement factor at each point is estimated as $1+X_{\gamma}^{c} / \chi_{\gamma}^{0}=1.3 \sim 2.5$ for $\gamma=x z$ and $x^{2}-y^{2}$ in the present calculation near the critical point. This effect will increase $(J / U)_{c}$ sensitively. The second factor is the $e$-ph interaction: We introduce the quadrupole interaction in Eq. (1) due to Fe-ion oscillations $[6,14,27]$. As shown in Fig. 3 (b), very strong AF-orbital fluctuations are obtained for $J / U=0.09$ and $g=0.05 ; \alpha_{\max }^{s}=\alpha_{0}^{c}=0.97$ and $\alpha_{\boldsymbol{Q}}^{c}=0.96$. The corresponding dimensionless coupling is just $\lambda=g N(0) \sim 0.035[6,27]$. We also study the case $n=6.05$ and $g=0.065$, and find that the relation $\alpha_{\max }^{s}=\alpha_{\max }^{c}=0.97$ is realized at $(J / U)_{c}=0.11$, as shown in Fig. 3 (c). For these reasons, strong ferro- and AF-orbital-fluctuations would be realized by the cooperation of the Coulomb and weak $e$-ph interactions.

Finally, we make some comments: The present multifluctuation mechanism is not described by the dynamicalmean-field theory (DMFT), since the irreducible VC is treated as local. Also, the local density approximation (LDA), in which the $\mathrm{VC}$ is neglected, does not reproduce the nonmagnetic orthorhombic phase. Although Yanagi et al. studied $U^{\prime}>U$ model [7] based on the RPA, that was first studied in Ref. [31], $\chi_{3 z^{2}-r^{2}}^{c}(\mathbf{0})$ develops while $\chi_{x^{2}-y^{2}}^{c}(\mathbf{0})$ remains small, inconsistently with the structure transition. Our important future issue is to include the electron self-energy correction into the SC$\mathrm{VC}$ method, which is important to discuss the filling and $T$-dependences of orbital and spin fluctuations, and to obtain more reliable $(J / U)_{c}$.

In summary, we developed the SC-VC method, and obtained the Coulomb-interaction-driven nematic and AF-orbital fluctuations due to the multimode (orbitons+magnons) interference effect [28] that is overlooked in the RPA. For $J / U \lesssim(J / U)_{c}$, the structure transition $\left(\alpha_{0}^{c} \approx 1\right)$ occurs prior to the magnetic transition $\left(\alpha_{\boldsymbol{Q}}^{s} \approx 1\right)$, consistently with experiments. When $\alpha_{\max }^{s} \sim \alpha_{\max }^{c}$, both $s_{++}$and $s_{ \pm^{-}}$states could be realized, depending on model parameters like the impurity concentration $[3,6]$. In a sense of the renormalization group scheme, the quadrupole interaction in Eq. (1) is induced by the Coulomb interaction beyond the RPA. We expect that orbital-fluctuation-mediated superconductivity and structure transition are realized in many iron-based superconductors due to the cooperation of the Coulomb and $e$-ph interactions.

This study has been supported by Grants-in-Aid for Scientific Research from MEXT of Japan, and by JST, TRIP. Part of numerical calculations were performed on the Yukawa Institute Computer Facility.

[1] K. Kuroki et al., Phys. Rev. Lett. 101, 087004 (2008).

[2] I. I. Mazin, D. J. Singh, M. D. Johannes, and M. H. Du, Phys. Rev. Lett. 101, 057003 (2008).

[3] P. J. Hirschfeld, et al., Rep. Prog. Phys. 74, 124508 (2011).

[4] A. V. Chubukov, arXiv:1110.0052.

[5] F. Wang et al., Phys. Rev. Lett. 102, 047005 (2009): The used parameters are $U=4, U^{\prime}=2$ and $J=0.7 \mathrm{eV}$.

[6] H. Kontani and S. Onari, Phys. Rev. Lett. 104, 157001 (2010).

[7] Y. Yanagi et al., Phys. Rev. B 81, 054518 (2010).

[8] M. Sato et al., J. Phys. Soc. Jpn. 79 (2009) 014710; S. C. Lee et al., J. Phys. Soc. Jpn. 79 (2010) 023702.

[9] Y. Nakajima et al., Phys. Rev. B 82, 220504 (2010).

[10] J. Li et al., Phys. Rev. B 84, 020513(R) (2011); J. Li et al., Phys. Rev. B 85, 214509 (2012).

[11] K. Kirshenbaum et al., arXiv:1203.5114.

[12] S. Onari and H. Kontani, Phys. Rev. Lett. 103177001 (2009).

[13] T. Shimojima et al., Sccience 332, 564 (2011).

[14] T. Saito et al. Phys. Rev. B 82, 144510 (2010).

[15] S. Onari et al., Phys. Rev. B 81, 060504(R) (2010); S. Onari and H. Kontani, Phys. Rev. B 84, 144518 (2011).

[16] R.M. Fernandes et al., Phys. Rev. Lett. 105, 157003 (2010).

[17] T. Goto et al., J. Phys. Soc. Jpn. 80, 073702 (2011).

[18] M. Yoshizawa et al., Phys. Soc. Jpn. 81, 024604 (2012).

[19] J.L. Niedziela et al., Phys. Rev. B 84, 224305 (2011).

[20] M. Yi et al., PNAS 1086878.

[21] Y. K. Kim et al., arXiv:1112.2243. 
[22] I. R. Fisher et al., Rep. Prog. Phys. 74124506 (2011).

[23] S. Kasahara et al., Nature 486, 382 (2012).

[24] Y. Inoue et al., Phys. Rev. B 85, 224506 (2012).

[25] C.-C. Lee et al., Phys. Rev. Lett. 103, 267001 (2009); W. Lv et al., Phys. Rev. B 82, 045125 (2010); K. Sugimoto et al., J. Phys. Soc. Jpn. 80 (2011) 033706.

[26] T. Miyake et al., J. Phys. Soc. Jpn. 79, 044705 (2010).

[27] S. Onari and H. Kontani, Phys. Rev. B 85, 134507 (2012).

[28] H. Kontani et al., Phys. Rev. B 84, 024528 (2011).
[29] T. Moriya, Spin Fluctuations in Itinerant Electron Magnetism (Springer-Verlag, 1985); A. Kawabata: J. Phys. F 4 (1974) 1477.

[30] N. E. Bickers and S. R. White Phys. Rev. B 43, 8044 (1991); K. Morita et al., J. Phy. Soc. Jpn. 72, 3164 (2003).

[31] T. Takimoto et al., J. Phys.: Condens. Matter 14, L369 (2002). 